\title{
La libération des neurotransmetteurs dans \\ le système nerveux central
}

La libération des neurotransmetteurs au niveau du système nerveux central est un phénomène discontinu «quantique », chaque quantum correspondant au contenu total d'une vésicule synaptique. La réponse post-synaptique dépend du nombre $n$ de zones actives (contacts fonctionnels entre l'afférence présynaptique et sa cible post-synaptique) et de la probabilité $p$ qu'un influx entraîne, au niveau de chacune des zones actives, l'exocytose d'une vésicule synaptique - et d'une seule. La plasticité de la synapse (facilitation ou dépression), dans différentes conditions, ne dépend que des variations du terme $p$.

\section{Henri Korn}

"Il y a quelques milliards d'années, une petite tache anonyme de protoplasme a fait saillie dans le pseudopodium primitif du limon primordial et dès lors survint le premier état d'incertitude ». Good, Science, 1959.

\section{ADRESSE}

H. Korn: Directeur de recherche à l'Inserm. Directeur de l'U.261 de l'Inserm. Laboratoire de neurobiologie cellulaire, Inserm U.261, département des biotechnologies, Institut Pasteur, 28, rue du Dr Roux, 75274 Paris Cedex 15, France. l existe aujourd'hui encore un paradoxe lié au fait que nos connaissances concernant les mécanismes par lesquels les neuromédiateurs sont émis, d'un neurone vers l'autre, restent limitées lorsqu'il s'agit des structures cérébrales profondes, alors que, à l'inverse, un nombre sans cesse croissant de neurotransmetteurs est découvert dans le système nerveux central (SNC), et que leur biochimie devient de plus en plus connue. Le principe de Dale suivant lequel un seul transmetteur peut être libéré par un neurone donné, est lui aussi remis en question par la découverte spectaculaire de la co-transmission associant au sein de mêmes terminales, acides aminés et peptides [1,2], voire plusieurs acides aminés [3]. Et pourtant le mode de survenue et le contrôle de la neurotransmission constituent des problèmes clés puisque, en dernière analyse, la transmission de l'information dans le cerveau s'effectue précisément par l'intermédiaire de ces substances. A ce paradoxe s'en ajoute un autre, mais apparent celui-là: la découverte des processus qui président à la libération des médiateurs passe par la connaissance et l'utilisation des lois du hasard, c'est-à-dire, si l'on y songe, des lois du jeu. A cet égard, les mécanismes de la neuromédiation constitueraient le meilleur argument en faveur de l'aphorisme de Laplace selon lequel « les questions les plus importantes de la vie sont, pour l'essentiel, des questions de probabilité». Rappelons ici que, loin d'être l'antithèse de toute loi, le hasard est gouverné au contraire par des lois mathématiques fixes et bien connues, dès qu'apparaissent en particulier les grands nombres. Que l'on remplace le terme de hasard, afin de rassurer le lecteur qui ne serait pas joueur, par celui de «statistiques», ne change rien au sens de ce message.

\section{Point de départ : la jonction neuromusculaire}

Pour des raisons techniques évidentes, nombre des aspects fondamentaux de la transmission synaptique ont été étudiés d'abord au niveau de jonctions directement abordables de visu. Tel est le cas de la jonction neuromusculaire: l'accès simultané des éléments pré- et post-synaptiques 


\section{A}

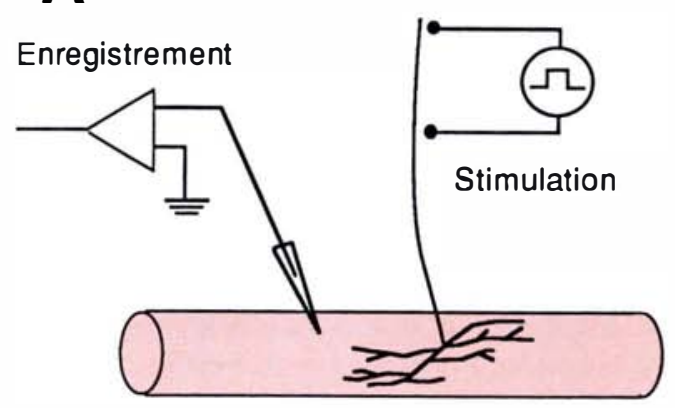

C

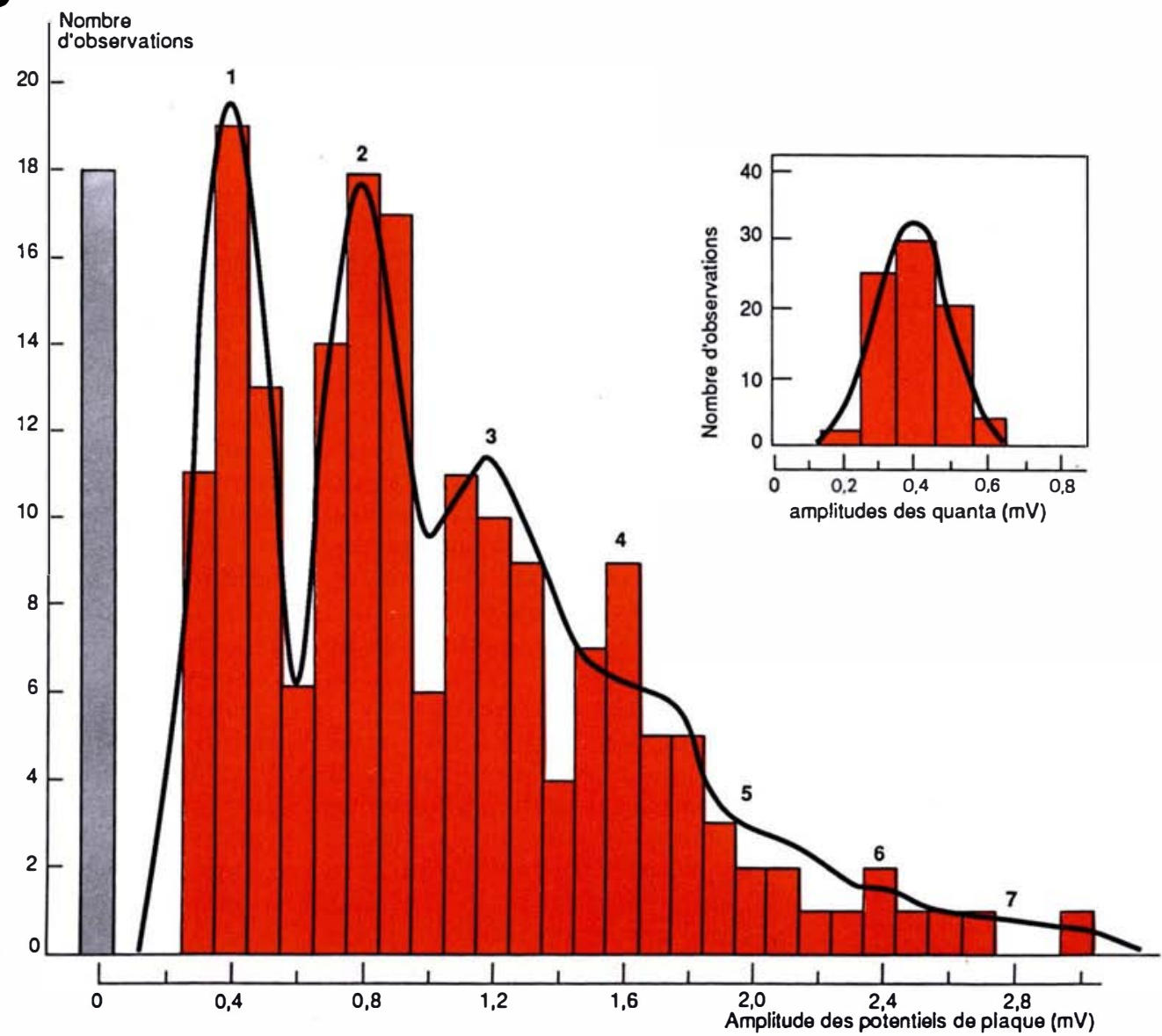

Figure 1. Mise en évidence du mode quantique de la libération à la jonction neuromusculaire. A. Principe d'enregistrement des réponses évoquées dans le muscle par l'activation du nerf afférent. B. Potentiels enregistrés qui peuvent être, soit spontanés et de faible amplitude mais toujours égale entre eux (quanta), soit évoqués par la stimulation et constitués dans ce cas par la sommation d'un nombre variable, d'un essai à l'autre, de ces potentiels miniatures. C. Distribution des potentiels évoqués par voie synaptique (zone rouge) constituée par des pics espacés de façon régulière, et ajustement de cet histogramme par une fonction calculée à partir des lois de Poisson (courbe noire). Les cas où la stimulation nerveuse a été efficace sont représentés en gris; ils permettent de calculer $m$ ou contenu quantique moyen (tiré de [5]). 


\section{RÉFÉRENCES}

1. Höckfelt T, L undberg JM, Schultzberg $M$, Johansson $\mathrm{O}$, Ljungdahl A, Rehfeld J. Coexistence of peptides and putative transmitters in neurons. In : Costa E, Trabucchi M, eds. Neural Peptides and Neuronal Com. munication. New York: Raven Press, 1980: $1-23$.

2. Höckfelt T Neuronal communication through multiple coexisting messengers. In : Edelman GM, Gall WE, Cowan WM, eds, Synaptic Function. New York: John Wiley and Sons Inc 1987: 179-211.

3. Triller A, Cluzeaud F, Korn H. Gammaamminobutyric acid-containing terminals can be apposed to glycine receptors at central synapses. J Cell Biol 1987 ; 104 : 947-56.

4. Katz B. The release of neural transmitter substance. Springfield: Charles C. Thomas, 1969.

5. Boyd IA, Martin AR. The end-plate potential in mammalian muscle. J Physiol (Lond) $1956 ; 132: 74-91$.

6. Kuffler SW, Yoshikami D. The number of transmitter molecules in a quantum : an estimate for iontophoretic application of acetylcholine at the neuromuscular synapse. J Physiol (Lond) 1975 ; 251, 465-82.

7. Katz B, Miledi R. The role of calcium in neuromuscular facilitation. J Physiol (Lond) 1968; 19: 481-92.

8. Llinas R, Steinberg IZ, Walton K. Presynaptic calcium currents and their relation to synaptic transmission. Voltage clamp study in giant synapse and theoretical model for the calcium gate. Proc Natl Acad Sci USA 1976 ; $73: 2918-22$.

9. Faber DS, Korn H. Binary mode of transmitter release at central synapses. Trends $\mathrm{Neu}$ rosci $1985 ; 5: 157-9$.

10. Edwards FR, Redman SJ, Walmsley B. Statistical fluctuations in charge transfer at Ia synapses on spinal motoneurones. $J$. Physiol (Lond) $1976: 259: 655-88$.

11. Triller A, Korn H. Déformations de la grille synaptique liées à l'exocytose dans les synapses centrales. CR Acad Sci (Paris) 1982: 295 : 655-60. de ces jonctions (figure $1 A$ ) permet de manipuler simplement les paramètres liés à la libération de transmetteurs (ici l'acétylcholine) lorsque l'influx qui s'est propagé le long de l'axone atteint les terminaisons de ce cable. Les données ainsi obtenues comptent parmi les plus solidement établies de la neurobiologie, et ont ainsi valu à B. Katz le prix Nobel de Médecine en 1970, alors même que la validité des lois qu'il avait élucidées restait pourtant à démontrer dans le cas du SNC. Celles-ci associent deux aspects [4, 5] illustrés dans la figure $1 B$ : (a) les réponses évoquées par la stimulation du nerf sont composées par la sommation, en nombre variable, de potentiels unitaires d'amplitude égale, d'où des fluctuations d'un essai à l'autre, du fait que 1,2 , $3 \ldots \mathrm{n}$ unités ont été libérées; (b) les potentiels spontanés enregistrés en l'absence d'influx sont semblables à ces unités de base, il s'agit donc de potentiels miniatures appelés pour cela quanta, par référence à ceux de la physique quantique. La mise en évidence du fait que les potentiels synaptiques sont ainsi composés d'unités discrètes, a reposé sur la confrontation de données expérimentales (distribution de leurs amplitudes) avec l'utilisation de lois mathématiques, ici de Poisson, qui prédisent que, à partir de la moyenne $m$ des paquets émis par un influx, on peut calculer la probabilité $p$, que surviennent, sur un grand nombre d'essais, $0,1,2,3 \ldots \times$ paquets, ou quanta, à partir de l'équation :

$$
\mathrm{p}_{(\mathrm{x})}=\mathrm{e}^{-\mathrm{m}} \mathrm{m}^{\mathrm{x}} / \mathrm{x} \text { ! ( } 1 \text { ) }
$$

On voit que le seul problème est de déterminer la valeur du paramètre $\mathrm{m}$, qui est appelé contenu quantique moyen, et deux méthodes sont pour cela à notre disposition.

(a) Dans des conditions où $\mathrm{p}$ est artificiellement réduit (calcium bas, Mg élevé dans le bain), on observe un grand nombre d'échecs, $\mathrm{p}_{0}$, c'est-àdire d'influx non suivis de libération, avec $\mathrm{p}_{(0)}=\mathrm{e}^{-\mathrm{m}}=$ (nombre d'échecs)/(nombre total d'influx), de sorte que $\mathrm{m}$ devient le logarithme de cette seule fraction.

(b) La seconde méthode repose sur le fait que $\mathrm{m}=$ (moyenne des réponses évoquées)/(amplitude moyenne des potentiels spontanés).

Dès lors, le nombre de potentiels constitués par $1,2,3 \ldots \times$ unités dis- crètes peut être prédit en cascade puisque, à partir du seul nombre d'échecs, $\mathrm{n}_{\hat{0}}$, un histogramme théorique peut être reconstruit, en calculant $\mathrm{n}_{0}=\mathrm{e}^{-\mathrm{n}} ; \mathrm{n}_{1}=\mathrm{mn}_{0} ; \mathrm{n}_{2}=$ $m / 2 . n_{1} ; n_{3}=m / 3 . n_{2}$, etc. La figure $1 C$, où sont comparées les distributions expérimentales des potentiels musculaires consécutifs à des stimulations répétées (rectangles) et théorique (courbes pleines), confirme la validité de ce modèle: les réponses évoquées sont représentées par une succession de pics, séparés entre eux par des intervalles égaux correspondant chacun à l'amplitude des potentiels spontanés observés dans la même préparation (encart).

\section{Nature vésiculaire des quanta}

Le quantum d'acétylcholine apparaît donc, au vu des données cidessus, comme relativement constant malgré les larges variations des conditions cellulaires qui, par ailleurs, en contrôlent la fréquence d'émission. D'autre part, le modèle de Poisson implique que chaque quantum libéré est choisi au hasard au sein d'une large population disponible $(\mathrm{n})$, chacun de ces éléments ayant une probabilité $\mathrm{p}$ très faible d'être choisi à chaque coup. Rappelons ici que le terme m est égal au produit np et qu'il définit le contenu quantique, ou quantité de transmetteur libéré en moyenne au niveau de la jonction synaptique lors de stimulations itératives. Il était donc tentant de supposer que le quantum représente le contenu en molécules de transmetteur stocké dans un des organelles, lui-même présent en quantité abondante dans la synapse, et dont la morphologie présente des caractéristiques constantes. Tel est le cas des vésicules synaptiques, sphères de 30 à $50 \mathrm{~nm}$ (nanomètres) de diamètre, présentes en larges quantités au voisinage de la synapse, et plus précisément de régions privilégiées de celle-ci, appelées zones actives à la jonction neuromusculaire, où s'effectuerait l'émission des quanta; chaque terminaison nerf-muscle contient 500 à 1000 de ces zones actives. La collision des vésicules avec la surface de la membrane axonale provoquerait l'ouverture de celles-ci, avec libération du contenu 

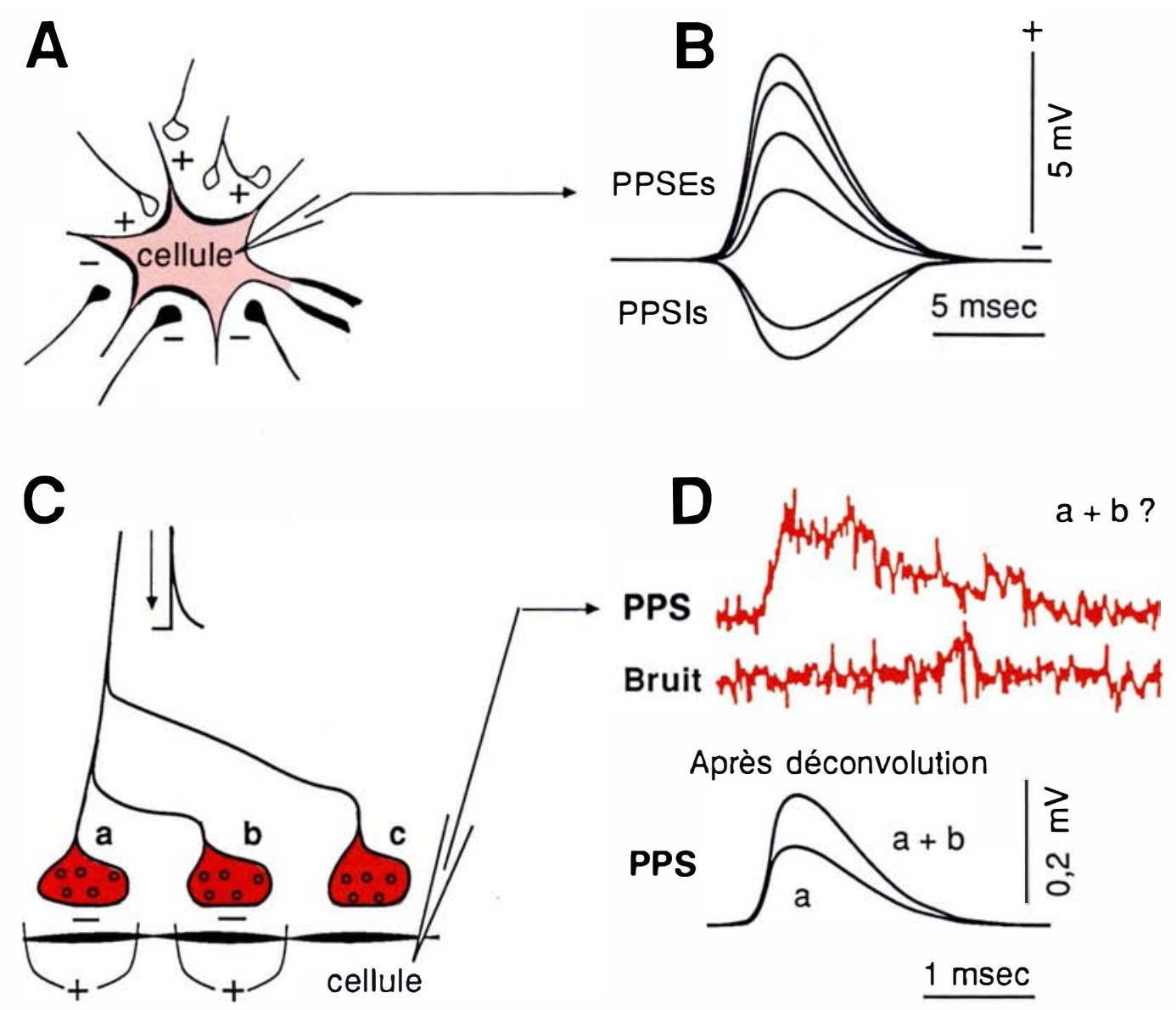

Figure 2. Difficultés rencontrées lors de l'analyse des potentiels synaptiques dans un neurone central. A. Polyinnervation d'un neurone qui est soumis en permanence à un bombardement à partir d'afférences excitatrices (t) et inhibitrices (-). B. Fluctuations spontanées dues à la sommation, aléatoire, de potentiels excitateurs (PPSEs) et inhibiteurs (PPSIs). C. Boutons terminaux établis par un neurone afférent sur sa cellule cible. Lorsque l'influx nerveux se propage dans le sens orthodromique (flèche), il provoque la libération de neurotransmetteur qui, après s'être lié aux récepteurs postsynaptiques, détermine l'apparition de courants et par conséquent de potentiels d'amplitude variable, dont il s'agit de déterminer la nature quantique (PPS = potentiel post-synaptique). D. Cette évaluation nécessite la mesure de l'amplitude des potentiels enregistrés qui est masquée par un bruit thermique; ceux-ci peuvent être néanmoins * lavés * par un procédé mathématique appelé déconvolution (tiré de [9]).

vésiculaire dans l'espace synaptique (exocytose). Des évaluations indirectes, électrophysiologiques, ont permis d'estimer à environ 10000 molécules d'acétylcholine [6] le contenu vésiculaire. Mais ce chiffre pourrait être de $10^{5}$ dans le ganglion parasympathique et des mesures directes, ainsi que la visualisation de mouvements éventuels de vésicules synaptiques lorsque le nerf est activé, restent encore un exploit technique hors de notre portée sur un matériel vivant.

Il est généralement admis que, au $\mathrm{m} / \mathrm{s} n^{\circ} 8$ vol. 4 , octobre 88 repos, un nombre restreint de vésicules s'ouvre au hasard, et de façon irrégulière, provoquant l'apparition des potentiels miniatures spontanés mentionnés ci-dessus. L'arrivée du potentiel d'action augmente de façon spectaculaire la probabilité des exocytoses : pendant la milliseconde que dure celui-ci, plusieurs centaines de quanta peuvent être émis simultanément à la jonction neuromusculaire. Ce fait est lié à la dépolarisation de la terminaison (l'influx s'accompagne d'une entrée de $\mathrm{Na}^{+}$dans la cellule) et celle-ci a pour consé- quence d'ouvrir, au niveau de la membrane, des canaux voltagedépendants, sélectifs aux ions $\mathrm{Ca}^{++}$ [7, 8], qui sont présents en quantité abondante autour de la zone active. Un nuage de calcium envahit brièvement la synapse et il provoque, par des mécanismes encore mal connus, la fusion des vésicules avec l'axolemme, et leur exocytose. Ce rôle du calcium est essentiel, sa concentration locale contrôle directement le contenu quantique, et plus généralement l'efficacité synaptique, dont les modifications, en plus ou en moins, 
sont à la base des effets de facilitation tels qu'on en observe immédiatement après un train d'influx, phénomène de potentiation post-tétanique lié à un résidu calcique persistant dans les terminales.

\section{Libération quantique dans le système nerveux central}

C'est depuis peu que la question a été abordée dans les structures centrales : comme l'illustre la figure $2 A$ $B$, les neurones centraux sont polyinnervés et, de plus, soumis à des influences contradictoires, excitatrices et inhibitrices, qui modifient dans un sens imprévisible [9], le potentiel de la membrane. Il n'est d'autre part pas aisé de reproduire les expériences de la jonction neuromusculaire qui supposent d'identifier deux neurones connectés entreeux, sauf dans des cas privilégiés, qui restent exceptionnels. La figure $2 C$ $D$ illustre une difficulté supplémentaire: les enregistrements des réponses évoquées par stimulation d'une fibre sont contaminés par le bruit de fond sans cesse présent dans la cellule, d'origine thermique et instrumentale, qui est aléatoire, et leur amplitude ne peut être évaluée, pour l'analyse de leurs fluctuations, qu'après un traitement mathématique destiné à les «laver» de ce bruit, appelé déconvolution [10].

Ces difficultés ont été surmontées au niveau des synapses inhibitrices d'un neurone situé dans le bulbe des poissons téléostéens, la cellule de Mauthner. Les cellules donnant naissance à ces synapses émettent un nombre variable, 3 à 60 , de boutons synaptiques caractéristiques illustrés par la figure 3 : on distingue, dans chacun d'eux, une zone active où peuvent être saisies des figures d'exocytose [11]. Le transmetteur libéré ici est la glycine [12] qui ouvre des canaux $\mathrm{C}^{-}$au niveau des récepteurs postsynaptiques [13] que l'on voit sur la figure marqués par des anticorps monoclonaux spécifiques [14].

Comme à la jonction neuromusculaire, ces neurones et leurs cibles ont été enregistrés simultanément et les histogrammes des réponses évoquées par la stimulation pré-synaptique ont été confrontés avec les prédic-

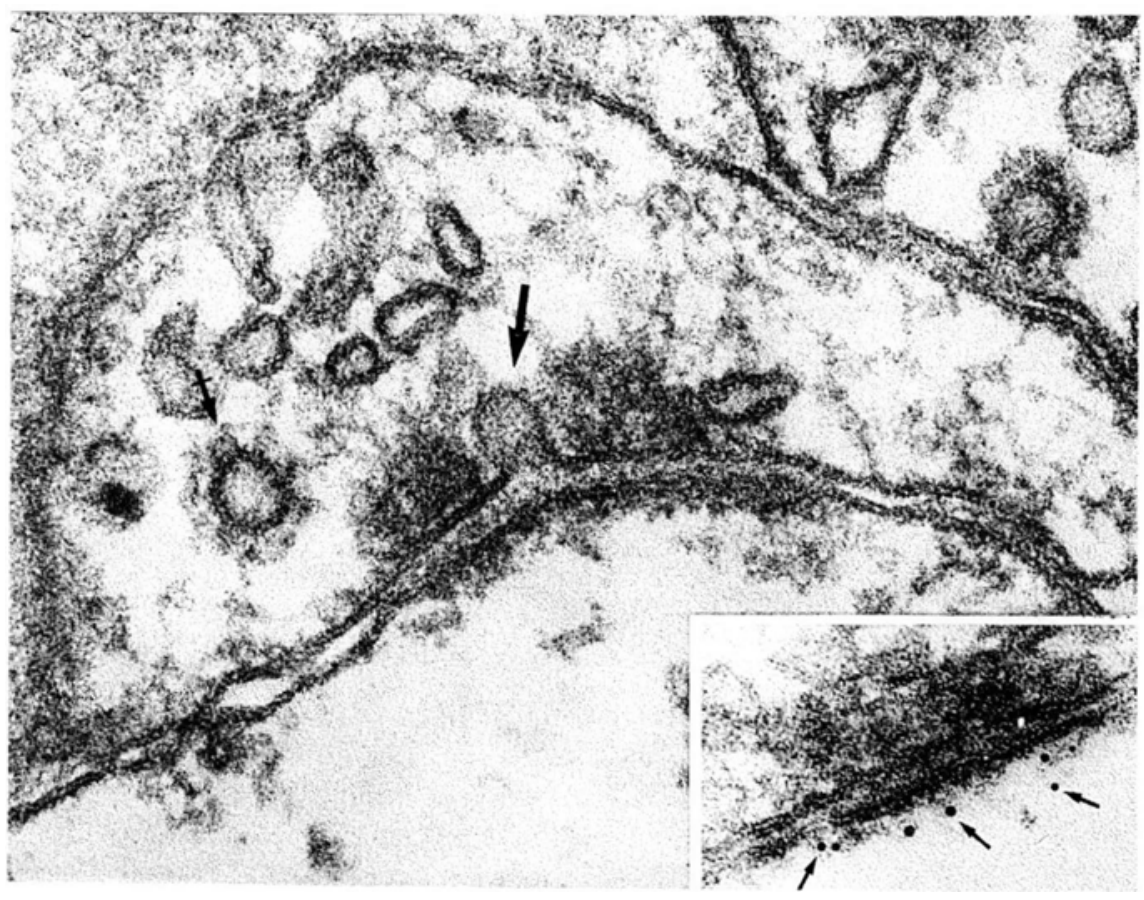

Figure 3. Synapse chimique caractéristique établie sur la cellule de Mauthner par un neurone inhibiteur. On note la présence de vésicules synaptiques (flèche barrée). L'une d'elles (flèche) a été saisie alors qu'elle déversait son contenu en neurotransmetteur dans la fente synaptique (exocytose). L'ouverture de cette vésicule s'est effectuée au niveau de la zone active caractérisée par la présence de densités qui «guident "les vésicules vers la membrane plasmique (tiré de [11]). Encart. Marquage des récepteurs post-synaptiques par des grains d'or colloïdal (flèches), eux-mêmes liés à un anticorps monoclonal spécifique dirigé contre les récepteurs à la glycine qui est le neuromédiateur de l'inhibition au niveau de certaines synapses centrales (tiré de [14]).

dérivé des lois du hasard, permettant de déterminer si les fluctuations de ces réponses étaient de nature discrète. La loi de Poisson, adaptée à des situations où $\mathrm{p}$, la probabilité d'exocytose, est faible, a dû être remplacée par le cas, plus général, des lois binomiales. Ce terme signifie simplement deux «noms», ou encore deux solutions, et il s'applique à des problèmes tels qu'il s'en présente lorsque l'on tire à pile ou face, par exemple. Si l'on a choisi pile et que l'on tire celui-ci, le premier terme est le succès et il survient avec une probabilité p, le second est l'échec (face), sa probabilité est $1-p$. Si l'on tire un grand nombre de fois, par exemple 10 fois, toutes les combinaisons sont possibles entre 0 et 10 fois pile, la plus probable étant cependant la moyenne, c'est-à-dire 5, du fait que les chances sont égales, c'est-àdire 1/2. Mais la loi binomiale permet de prédire plus précisément quelle sera la répartition des évènements, c'est-à-dire la chance de tirer pile un nombre donné de fois, par exemple 3 ou 8 , sur un grand nombre de coups, pour une probabilité p donnée. En prenant un autre exemple, celui du jeu des dés, la probabilité de tirer 6 en un seul coup, sera de $1 / 6$, celle d'échouer sera de $5 / 6$, sur un nombre élevé d'essais. Il existe néanmoins des chances non négligeables de tirer un chiffre différent de cette moyenne théorique. En définitive, l'histogramme des réponses sera, selon toute vraisemblance, biaisé, la fréquence des succès étant moindre que celle des échecs.

Les distributions des réponses synaptiques sont effectivement bien ajus- 
A

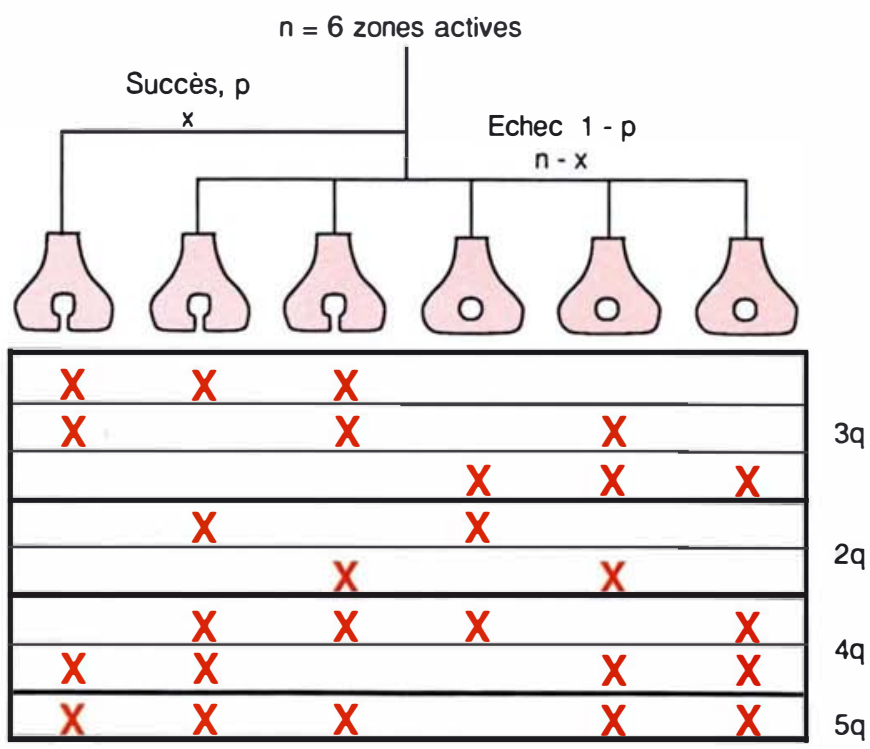

Amplitude des réponses

après chaque stimulation

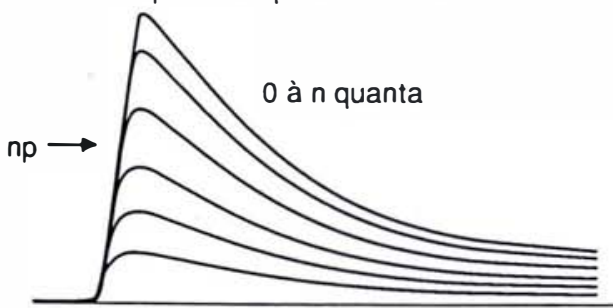

$3 q$

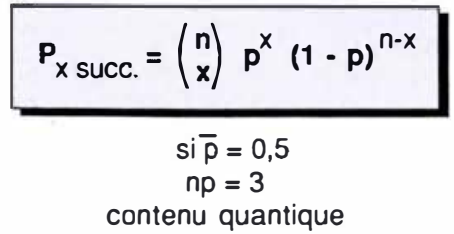

Figure 4. Signification du mode binomial de la libération de neuromédiateur. A. Schéma d'un neurone se terminant par six boutons synaptiques. Après chaque influx, un nombre variable $x$ de ces boutons émet le neurotransmetteur (succès, dont la probabilité est p), d'autres restent inactifs (échecs, dont la probabilité est 1-p). Les cas suivis d'exocytose sont représentés avec des vésicules ouvertes. Dans le cas choisi, $p$ est égal à 0,5 , de sorte qu'une moyenne de trois boutons sera activée mais avec une combinaison variable de ceux-ci; les exocytoses surviennent de façon aléatoire lors de stimulations successives dont l'issue est représentée, sous forme de croix, dans les lignes horizontales. Noter cependant que dans un certain nombre d'essais, 2, 4 ou 5 d'entre elles sont observées, bien qu'en proportion moindre. B. Fluctuation des réponses post-synaptiques déterminée par le nombre de quanta qui les constituent. Etant donné que le contenu quantique (np, flèche) est ici de 3, les potentiels correspondant à cette amplitude seront les plus fréquents. La formule binomiale qui permet de calculer la probabilité $p(x)$ qu'apparaissent 1, 2, 3... x quanta montre que $n$ et $p$ sont distincts dans cette équation.

tées par des fonctions binomiales [15, 16] qui ont confirmé que la libération est quantique dans le système nerveux central. Confrontées avec les données de la morphologie, elles ont donné des indications précieuses sur la manière dont les terminaisons d'un même neurone fonctionnent alternativement, lors d'influx successifs. Ces conclusions sont illustrées sur la figure 4, qui permet de mieux comprendre également ce que sont les deux termes des équations binomiales. Le neurone schématisé ici donne naissance à 6 boutons synaptiques, c'est-à-dire à autant de zones actives. Après chaque potentiel d'action, toutes les combinaisons sont possibles, entre la libération de médiateur au niveau de $1,2 \ldots 6$ terminaisons, et si la probabilité moyenne d'exocytose est de 0,5 , on aura, sur un grand nombre de coups, une prédominance de cas où 3 quanta sont émis et ce, par des combinaisons variables de boutons actifs. On retrouve le contenu quantique moyen, produit de $\mathrm{n}$ (égal ici à 6) et p (qui, dans le cas choisi, est de 0,5$)$. Il est intéressant de noter que dans l'équation utilisée, les termes $n$ et $\mathrm{p}$ apparaissent de façon distincte, alors que dans la loi de Poisson, seul apparaît leur produit $\mathrm{m}$. De ce fait, il a été possible d'assigner au paramètre $n$, qui jusque là était un paramètre mathématique abstrait, une réalité physique: il correspond au nombre de zones actives, c'est-à-dire de synapses établies par chaque neurone sur sa cellule cible [15, 16]. En d'autres termes, l'unité quantique correspond à la quantité de transmetteur émise, par bouton et par influx, cette quantité étant la même au niveau de chaque terminaison. Chacune d'entre elles fonctionne de façon indépendante de ses voisines, et de manière alternée, puisque $p$, qui d'un neurone à l'autre varie entre 0,2 et 0,6 , ne permet pas à toutes d'émettre de façon simultanée.

La flexibilité de p est à la base de la plasticité synaptique à court terme : on comprend aisément que toute variation de ce paramètre modifie dans le même sens le contenu quantique, et qu'il constitue ici la seule variable, dans la mesure où n, nombre de zones actives, et q, taille du quantum, sont des invariants structuraux du neurone, du moins chez l'adulte. Cette notion a été vérifiée par l'analyse binomiale des réponses induites dans la cellule cible lors de stimulations de neurones afférents 


\section{RÉFÉRENCES}

12. Faber DS, Korn H. Single-shot channel activation accounts for duration of inhibitory postsynaptic potentials in a central neuron. Science $1980 ; 208$ : 612-5.

13. Faber DS, Funch PG, Korn H. Evidence that receptors mediating central synaptic potentials extend beyond the postsynaptic density. Proc Natl Acad Sci USA 1985; 82 : 3504-8.

14. Triller A, Cluzeaud F, Pfeiffer F, Beu $H$, Korn $\mathrm{H}$. Distribution of glycine receptors at central synapses: an immunoelectron microscopy study. J Cell Biol $1985 ; 101: 683-8$.

15. Korn H, Triller A, Mallet A, Faber DS. Fluctuating responses at a central synapse : $\mathbf{n}$ of binomial fit predicts number of stained presynaptic boutons. Science 1981; 213 : 898901.

16. Korn H, Mallet A, Triller A, Faber DS Transmission at a central synapse. II - Quan tal description of release with a physical correlate for binomial n. J Neurophysiol $1982 ; 48: 679-707$.

17. Korn H, Faber DS, Brunod Y, Triller A. Regulation of efficacy at central synapses. $J$ Neurosci $1984 ; 4: 125-30$.

18. Eccles JC. The physiology of synapses. Berlin : Springer Verlag, 1964.

19. Faber DS, Korn H. Transmission at a central inhibitory synapse. I. Magnitude of the unitary postsynaptic conductance change and kinetics of channel activation. $J$ Neurophysiol $1982 ; 48: 654-78$.

20. Korn H, Burnod Y, Faber DS. Spontaneous quantal currents in a central neuron match predictions from binomial analysis of evoked responses. Proc Natl Acad Sci USA $1987 ; 84: 5981-5$.

21. Shannon CE, Weaver W. The mathematical theory of communication. Urbana : University of Illinois Press, 1949.

22. Korn H, Faber DS. Regulation and significance of probabilistic release mechanisms at central synapses. In : Edelman GM, Gall WE, Cowan WM, eds. Synaptic Function. New

York: Neuroscience Research Foundation

\section{A}

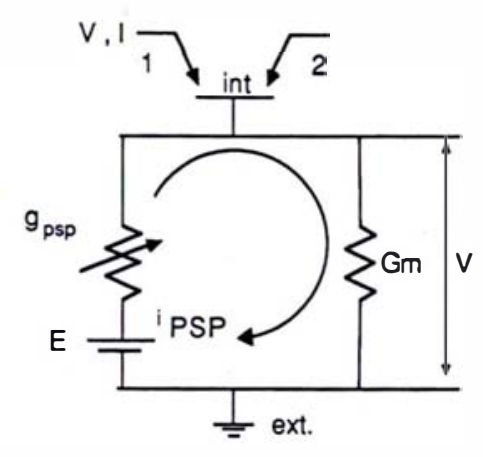

B

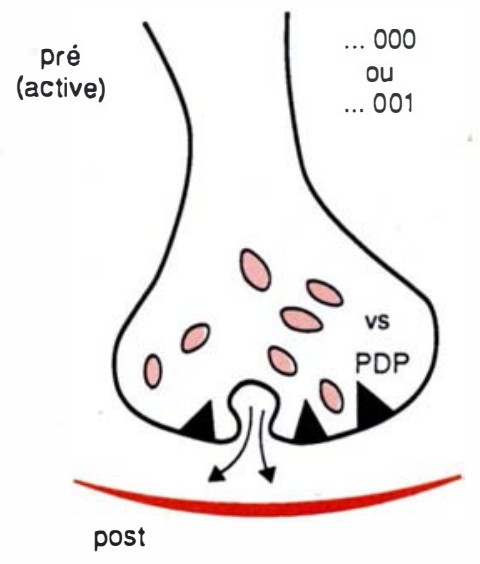

Figure 5. Circuit équivalent de la membrane post-synaptique et représentation graphique de la notion suivant laquelle un quantum représente l'effet du neurotransmetteur contenu dans une seule vésicule synaptique. A. Schéma indiquant que la réponse à l'activation d'une zone active est due à une augmentation de conductance $\left(g_{\text {pso }}\right.$ ) qui permet d'évaluer le nombre de canaux ioniques ouverts par le neuromédiateur. Cette résistance est en série avec une batterie $E$ représentant la force électromotrice de cet ion; le courant qui en résulte polarise ( $i_{\text {pso }}$ la membrane adjacente qui est représentée par sa conduction d'entrée (Gm) (d'après [19]). B. la terminaison pré-synaptique (pré) contient des vésicules synaptiques NS) et des zones de densité marquée (PDP) entre lesquelles survient l'exocytose lorsqu'un potentiel d'action dépolarise la terminale. Le transmetteur diffuse en direction de la membrane post-synaptique (post). Noter la représentation binaire qui souligne que, malgré la présence de nombreuses vésicules synaptiques, une seule d'entre elles peut, au maximum, libérer son contenu après un potentiel d'action (d'après [9] et [16]).

à des fréquences de plus en plus élevées [17]. Ce «tétanos» s'accompagne d'un phénomène de dépression post-synaptique constitué par une diminution d'amplitude des réponses; les paramètres $n$ et $q$ restent effectivement inchangés alors que la probabilité d'exocytose diminue de façon exponentielle en relation avec la fréquence de stimulation. Il faut noter ici que ce cas n'est pas général: dans certains systèmes, tel le motoneurone spinal, c'est au contraire une facilitation qui peut être induite dans de telles conditions expérimentales [18]. Mais là encore, le facteur $p$, qui cette fois évolue en sens inverse du cas précédent, est à incriminer, au moins en partie. Il semble que l'apparition d'une facilitation ou d'une dépression soit conditionnée par l'interaction de deux processus indépendants qui sont la concentration intracellulaire en calcium et la valeur initiale de $p$ qui, au contraire du terme $\mathbf{n}$ (nombre de zones actives), n'a pas encore de contrepartie physique clairement élucidée.

\section{Quanta de transmetteur et théorie de l'information}

Implicite dans le fait que la distribution d'amplitude des réponses sỵnaptiques est ajustée par une fonction binomiale, est la notion suivant laquelle la quantité de transmetteur libéré par une zone active est égale à celle issue par les autres synapses du neurone mis en jeu. Ce fait a été confirmé par l'analyse des quanta inhibiteurs de la cellule de Mauthner, qui sont égaux entre eux, ainsi que par des calculs faisant intervenir un circuit équivalent de la membrane [19] dont une simplification est montrée sur la figure $5 \mathrm{~A}$. Deux conductances (inverses de la résistance) y sont représentées en paral- 
lèle: $G m$, qui est celle mesurée au repos, et $g_{\text {psp }}$ qui est directement proportionnelle au nombre de canaux $\mathrm{Cl}^{-}$ouverts dans la membrane lorsqu'une synapse est active. Cette dernière est en série avec une batterie $\mathrm{E}$ représentant la force électromotrice développée par les ions $\mathrm{Cl}^{-}$lorsqu'ils passent à travers la membrane. Comme le montre la figure 5 , ces différents termes ont été évalués de façon directe à l'aide de micro-électrodes intracellulaires. Sans entrer dans le détail, on peut dire ici que la conductance liée à l'activation d'une zone active peut être calculée à partir de la relation $g_{q}=(q / E) G m$ qui a indiqué que le neuromédiateur libéré par une terminaison ouvre en moyenne l 000 canaux au niveau de la membrane post-synaptique [16, 20]. Ce chiffre est constant pour une cellule donnée et il correspond, selon les estimations faites par ailleurs à la jonction neuromusculaire, à l'effet d'une vésicule synaptique et d'une seule. Ainsi la théorie vésiculaire retrouve droit de cité dans le cas des synapses du SNC.

Il devient donc possible de représenter ce qui se passe au niveau d'une zone active lorsque la terminaison nerveuse est dépolarisée par un influx. Nous avons vu que celle-ci libère, de façon tout ou rien, et avec une certaine probabilité, un contenu invariant de médiateur qui, selon toute vraisemblance, correspond au maximum à celui d'une vésicule synaptique et d'une seule. Ce comportement binaire, représenté sur la figure $5 B$, s'apparente à celui, binaire également, de chacune des unités de base qui constituent un ordinateur. Sans entrer dans des analogies trop faciles, on peut rappeler que tout système de communication, tel qu'il a été schématisé par Shannon et Weaver [21], est constitué par cinq éléments : (1) une «source d'informations » qui, dans le système nerveux, serait représentée par l'axone présynaptique et ses ramifications; celui-ci «choisit» le message désiré et sa représentation physique dans le système nerveux serait précisément le contenu quantique np. (2) Une «source émettrice» transformant ce message en signal, et à cet égard la zone active serait un candidat approprié, d'autant que le choix tout ou rien d'un quantum est bien adapté pour que soit définie la quantité d'information transmise en utilisant l'unité classique du bit (binary digit). Cette quantité d'informations peut être calculée simplement par une formule qui ne fait intervenir que la probabilité de survenue d'un message [22]. Or nous savons que cette probabilité peut être définie par la loi binomiale. Pour un neurone comportant 16 terminaisons et une valeur de $p=0,5$, l'entropie, ou quantité d'informations à la source, est de 3,5 par zone active lorsque le neurone est stimulé à la fréquence de $1 /$ seconde. Pour des fréquences plus rapides de $30 /$ seconde, cette valeur atteint 2,5 bits. D'une façon générale, ce qui est intéressant ici, c'est que si le nombre de terminaisons émises par un neurone est faible, la quantité d'information contenue par un quantum est notable mais est particulièrement sensible aux modifications de p, c'est-àdire à l'état fonctionnel du neurone. L'inverse se produit dans le cas de neurones ayant un grand nombre de terminaisons ; ils transmettent moins d'informations par zone active mais ils garantissent par leur insensibilité aux variations de $\mathrm{p}$, une réponse fixe post-synaptique minimale. La structure compense ici les limites de l'organisation synaptique. (3) L'«élément transmis » d'une cellule à l'autre est la quantité de neurotransmetteur contenu dans les vésicules synaptiques, c'est le véritable messager de l'information qui va être reçu par (4) «le receveur » que l'on peut représenter par la membrane synaptique et ses récepteurs, et (5) le «destinataire» de ce message ou neurone post-synaptique dont le rôle est d'intégrer l'ensemble des signaux qui lui parviennent continuellement par l'intermédiaire de ses afférences excitatrices et inhibitrices.

Conçue dans ces termes, la transmission synaptique devient un mécanisme infiniment souple et variable, et cette plasticité qui est liée au caractère probabiliste de la libération de neurotransmetteurs [17], lui assure une flexibilité et une infinité de modes opératoires qui n'étaient en rien prévisibles dans la notion initiale, déterministe, de l'arc réflexe de Sherrington, point de départ de la neurobiologie contemporaine

\section{Summary}

Although the quantal aspect of transmitter release has been firmly established for a long time, evidence that such is also the case in the central nervous system has only been obtained recently. They are primarily based on the observation that postsynaptic responses produced by a single presynaptic neuron fluctuate in integer steps, the maximum of which corresponds to the number of active zones issued on its target by this presynaptic afferent. The release of transmitter becomes a probabilistic phenomenon characterized by a statistical parameter, $p$, which refers to the chance that each of these morphologically well-defined regions allows one synaptic vesicle to undergo exocytosis after a presynaptic impulse. This parameter is a variable term and its modifications solely account for the plasticity of the synapse during postsynaptic depression or facilitation under different physiological modifications of network activation. The notion that a quantum of transmitter is released in an allor-none or binary manner suggests that chemical transmission is more critically involved in the process of information transfer than previously suspected and that the input-output relation at central synapses is one of several processes embedded in Shannon's encoding and decoding schemes that might operate in the central nervous system.

TIRÉS A PART

H. Korn. 